\title{
Rendements et pratiques des cultures maraîchères en agriculture biologique au Sénégal
}

\author{
Hubert de Bon ${ }^{1,4, *}$, Laure Brun-Diallo ${ }^{2}$, Jean-Michel Sène ${ }^{2}$, Serge Simon ${ }^{1,3}$ et Mamadou Abdoulaye \\ Sow $^{2}$ \\ ${ }^{1}$ CIRAD, UPR HORTSYS, 34398 Montpellier, France \\ 2 ENDA-PRONAT, BP 3370, Dakar, Sénégal \\ 3 ISRA-CDH, Cambérène, Dakar, Sénégal \\ ${ }^{4}$ HORTSYS, Univ. Montpellier, Montpellier, France
}

Résumé - Les rendements en agriculture biologique font l'objet de controverses et sont considérés comme plus faibles qu'en agriculture conventionnelle. En Afrique, l'agriculture biologique recouvre une grande diversité de pratiques et les rendements présentent de fortes incertitudes. Les analyses des rendements et des itinéraires techniques en agriculture biologique sont présentées pour trois cultures maraîchères au Sénégal. Les résultats sont ensuite comparés aux rendements usuels de l'agriculture conventionnelle. Deux types de données sont utilisés, provenant de parcelles d'agriculteurs : i) suivi de champs conduits selon des itinéraires techniques d'agriculture biologique vs. itinéraires techniques conventionnels ; ii) résultats issus des actions de l'organisation non gouvernementale ENDA-PRONAT, suivant un cahier des charges copié sur celui de l'agriculture biologique. Les suivis comparatifs de parcelles de chou pommé et de tomate en saison des pluies mettent en évidence des ratios de rendement agriculture biologique/conventionnelle de 74 et $40 \%$ respectivement. Ces valeurs confirment la tendance générale des résultats de la littérature. Elles sont expliquées par une fertilisation azotée plus faible et l'absence de mesures de protection efficace contre les maladies et ravageurs des cultures. Les rendements en oignon des parcelles d'agriculture biologique chez les producteurs sont très variables: en moyenne de 19,4 t/ha à 11,0 t/ha suivant les années dans la zone des Niayes, 21,0 t/ha dans le Bassin arachidier et seulement 8,6 t/ha dans la vallée du fleuve Sénégal. Les rendements de la culture d'oignon au Sénégal observés dans la littérature varient aussi dans de grandes proportions, de 10,7 à $32 \mathrm{t} / \mathrm{ha}$. Dans certains cas, les rendements en agriculture biologique sont supérieurs à ceux des itinéraires techniques de l'agriculture conventionnelle. Ce constat, déjà observé dans la littérature scientifique, montre que l'agriculture biologique est une voie de développement de l'agriculture à ne pas écarter en Afrique.

Mots clés : Afrique subsaharienne / oignon / tomate / chou pommé / fertilisation organique

\begin{abstract}
Organic vegetable cropping systems yields and practices in Senegal. Yields in organic farming are controversial and are considered to be lower than in conventional agriculture. In Africa, organic farming practices are very diverse and yields are highly uncertain. Analyses of yields and crop management practices are described on three vegetable crops in Senegal. The results are then compared with the usual yields of conventional agriculture. Two types of data are used, all of which coming from farmers' fields: (i) monitoring of fields cultivated according to organic vs. conventional farming methods; (ii) results from the actions of the non-governmental organization ENDA-PRONAT following specifications copied from organic farming requirements. Comparative monitoring of cabbage and tomato fields in the rainy season showed organic/conventional farming yield ratios of 74 and $40 \%$ respectively. These values confirm the general trend of literature. They are explained by a lower nitrogen fertilization and lack of effective measures to protect crops against diseases and pests. The onion yields of organic farmers' fields are very variable: on average, from $19.4 \mathrm{t} / \mathrm{ha}$ to $11.0 \mathrm{t} / \mathrm{ha}$ depending on the years in the Niayes area, $21.0 \mathrm{t} / \mathrm{ha}$ in the Groundnut Basin and only $8.6 \mathrm{t} / \mathrm{ha}$ in the Senegal River Valley. According to scientific literature, yields of
\end{abstract}

\footnotetext{
*Auteur correspondant : hubert.de_bon@cirad.fr
} 
onion crops in Senegal also vary in large proportions, from 10.7 to $32 \mathrm{t} / \mathrm{ha}$. In some cases, yields in organic farming are higher than those in conventional farming. This observation, already noticed in the academic literature, shows that organic farming is a pathway for agriculture development not to be discarded in Africa.

Keywords: Sub-saharan Africa / onion / tomato / cabbage / organic fertilization

\section{Introduction}

Au Sénégal, les produits issus de l'agriculture biologique certifiée sont très divers : des céréales, des plantes oléagineuses (sésame, arachide), de l'oseille de Guinée (Hibiscus sabdariffa), mais aussi des fruits et légumes frais (mangue, oignon, tomate, pastèque). L'organisation non-gouvernementale (ONG), Environnement et développement du tiers-monde - Protection naturelle, ENDA-PRONAT, s'investit depuis trois décennies dans le développement de l'agriculture biologique (AB) aussi bien pour le marché national que pour le marché d'export.

L'ENDA-PRONAT a contribué à l'écriture du cahier des charges national de l'agriculture biologique au Sénégal, en tant que membre fondateur de la Fédération nationale de l'agriculture biologique (FENAB) en 2009. Cependant, à ce jour, le cahier des charges national est toujours en discussion avec l'International Federation of Organic Agriculture Movements (IFOAM). Pour le marché local, ENDA-PRONAT, afin d'éviter les conflits possibles sur l'appellation «agriculture biologique», a développé un cahier des charges spécifique pour une «agriculture saine et durable». Ce cahier des charges précise les pratiques agroécologiques dans les champs, les opérations post-récolte et la mise en marché. Il met l'accent sur la durabilité de l'agriculture en prenant en compte les caractéristiques du milieu, l'organisation des producteurs et la commercialisation des produits. Les principales recommandations en sont récapitulées dans le Tableau 1. Au total, ces règles nous permettent de considérer l'agriculture saine et durable comme agriculture biologique $(\mathrm{AB})$ dans la suite de cet article. Les résultats des actions techniques de l'ONG ont été rassemblés en 2016 sous forme de fiches de capitalisation sur l'agriculture écologique et biologique au Sénégal (ENDAPRONAT, 2016b). Cependant, malgré l'intérêt croissant de la certification biologique mise en place, seulement $11 \%$ de la production totale en agriculture biologique certifiée a été vendue comme telle (Tab. 2).

La question du rendement en agriculture biologique a fait l'objet de plusieurs méta-analyses récentes; elles ont mis en évidence des baisses de rendement dues à la pratique de l'agriculture biologique par rapport à l'agriculture conventionnelle (AC) utilisant engrais et pesticides de synthèse. Seufert et al. (2012), Ponisio et al. (2015) et Lesur-Dumoulin et al. (2017) ont essayé de rechercher les causes de ces baisses de rendement. Les variables suivantes ont été citées : apport azoté, $\mathrm{pH} \mathrm{du}$ sol, nature de l'engrais organique, irrigation, certification, durée depuis la conversion. Les systèmes de polyculture et les rotations culturales réduisent les pertes de rendement. Ce dernier élément est confirmé par ThorupKristensen et al. (2012) au Danemark. Mais Lesur-Dumoulin et al. (2017) ne montrent pas d'effets dus aux types de culture, aux zones climatiques, à la durée des expérimentations sur la variabilité du ratio $\mathrm{AB} / \mathrm{AC}$. Au-delà de ces contradictions, Kirchmann et al. (2016) soulignent quelques critères pour améliorer l'interprétation des méta-analyses: des niveaux initiaux de fertilité des sols similaires; des catégories de culture comparables; une quantification des matières organiques issues d'autres exploitations agricoles. Par ailleurs, Seufert et al. (2012) ont observé que les rendements en agriculture biologique des pays en développement étaient bien inférieurs $(-43 \% / \mathrm{AC})$ à ceux des pays développés $(-20 \% /$ AC), mais Ponisio et al. (2015) n'ont pas trouvé de telles différences de rendement. Cependant, les données issues des pays de l'Afrique sub-saharienne sont inexistantes. Un seul pays africain a été mentionné : la Tunisie dans Lesur-Dumoulin et al. (2017), Ponisio et al. (2015) et Seufert et al. (2012). Pour les cultures maraîchères, il n'apparaît pas de différence statistique pour Ponisio et al. (2015) entre AB et AC, alors que Seufert et al. (2012) calculent une baisse de rendement statistiquement significative de $-33 \%$. Lesur-Dumoulin et al. (2017) calculent que les rendements en horticulture (racines et tubercules, maraîchage, fruits, épices) sont en moyenne de 10 à $32 \%$ plus faibles en agriculture biologique, avec une baisse moyenne de $21 \%$ pour les cultures maraîchères par rapport aux systèmes conventionnels. Ils calculent que les pertes de rendement ont $10 \%$ de chances d'excéder $50 \%$ de celles des systèmes conventionnels. Mais ils montrent qu'il y a $20 \%$ de chances d'obtenir des rendements plus élevés. Toutes ces données proviennent essentiellement de stations expérimentales.

Notre étude vise à préciser les potentialités des cultures maraîchères en agriculture biologique en Afrique subsaharienne, en milieu paysan. L'objectif est de mesurer la variabilité des rendements en agriculture biologique et de quantifier les rendements des cultures maraîchères paysannes dans le système d'agriculture biologique par rapport au conventionnel. Nous utiliserons deux sources de données :

- des suivis de parcelles maraîchères en milieu paysan;

- les rapports d'activités de l'ONG ENDA-PRONAT (ENDA-PRONAT, 2016a, 2016b) pour répondre à nos objectifs sur les cultures de l'oignon, du chou pommé et de la tomate au Sénégal.

\section{Matériel et méthode}

\subsection{Revue de littérature}

La revue de littérature vise à identifier les articles présentant des données de rendements de chou pommé, tomate et oignon au Sénégal en milieu paysan.

\subsection{Contextes pédoclimatiques}

Les données proviennent de trois zones du Sénégal (Fig. 1) :

- la moyenne vallée du fleuve Sénégal pour la commune de Guédé (au nord du Sénégal), avec une pluviométrie de 200 
Tableau 1. Recommandations de pratiques d'agriculture biologique d'après le cahier des charges de l'agronomie saine et durable d'ENDAPRONAT.

Table 1. Recommendations of organic agriculture practices from the ENDA-PRONAT specifications of the sustainable and healthy agronomy.

\begin{tabular}{ll}
\hline Critères & Agriculture saine et durable (ou biologique) (AB) \\
\hline Délai de conversion & Un an
\end{tabular}

Gestion du paysage

Distance de $15 \mathrm{~m}$ avec parcelles en agriculture conventionnelle (AC)

Gestion du paysage Respect des caractéristiques du milieu (flore, ressources naturelles...) Respect de la diversité des éléments du paysage

Rotation des cultures

Engrais chimiques interdits

Engrais minéraux d'origine naturelle

Fertilisation

Fumiers et composts (végétaux et animaux), litière de volaille (sauf celles provenant d'élevage hors-sol), déchets ménagers compostés, boues d'épuration, coques d'arachide

Engrais verts

Semences

Protection contre les ravageurs et maladies

Organisation des producteurs
Semences OGM interdites

Pesticides de synthèse interdits

Protection physique, préparations naturelles à base de plantes, biopesticides

Contrôle des pratiques par un technicien et un membre du comité de suivi. Le non-respect du cahier des charges entraîne un déclassement

de la parcelle, de la production ou du producteur

Tableau 2. Productions de l'agriculture biologique en 2013 au Sénégal d'après les données IFOAM FiBL transmises par Famara Diedhiou: données totales et pour quelques cultures vivrières et horticoles.

Table 2. Organic agriculture productions in 2013 in Senegal from FOAM FiBL data transmitted by Famara Diedhiou: global data and for some food and horticultural crops.

\begin{tabular}{|c|c|c|c|c|c|}
\hline & $\begin{array}{l}\text { Superficie } \\
\text { en conversion (ha) }\end{array}$ & $\begin{array}{l}\text { Superficie } \\
\text { certifiée (ha) }\end{array}$ & $\begin{array}{l}\text { Superficie } \\
\text { totale (ha) }\end{array}$ & $\begin{array}{l}\text { Production } \\
\text { (t) }\end{array}$ & $\begin{array}{l}\text { Production vendue } \\
\text { comme certifiée }(\mathrm{t})\end{array}$ \\
\hline Toutes cultures & 4842 & 2087 & 6292 & 11478 & 1278 \\
\hline Sorgho & 47 & & 47 & 33 & \\
\hline Fonio & & 113 & 113 & 101 & 76 \\
\hline Sésame & 277 & 810 & 1087 & 652 & 500 \\
\hline Coton & & 100 & 100 & 17 & 17 \\
\hline Hibiscus sabdariffa & 158 & & 158 & 9 & \\
\hline Tomate & 30 & & 30 & 750 & \\
\hline Pastèque & 20 & 12 & 32 & 640 & 160 \\
\hline Mangue & 103 & 587 & 690 & 1034 & 206 \\
\hline
\end{tabular}




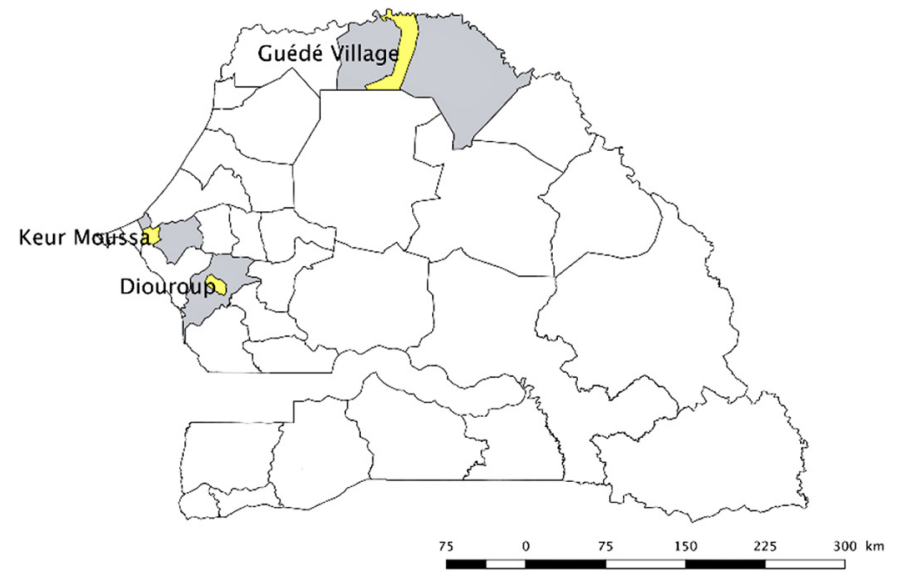

Fig. 1. Localisation des trois zones d'études au Sénégal (en gris).

Fig. 1. Location of the three study areas in Senegal (grey).

à $250 \mathrm{~mm}$ et un climat sahélien avec une saison sèche très marquée;

- l'ouest du bassin arachidier pour la commune de Diouroup, avec un climat sahélien plus humide $(500 \mathrm{~mm})$;

- la zone des Niayes pour la commune de Keur Moussa, avec un climat de type sub-canarien et une pluviométrie de $400 \mathrm{~mm}$.

La saison des pluies s'étend de juillet à octobre. L'eau d'irrigation nécessaire aux cultures provient du fleuve Sénégal dans la vallée et des nappes phréatiques dans les Niayes et le bassin arachidier. La préparation du sol est manuelle ou mécanisée. Le désherbage est manuel. Les épandages des matières organiques et des engrais sont faits manuellement, ainsi que les applications de produits de protection phytosanitaires.

Les sols des Niayes sont de type ferrugineux tropical faiblement lessivé sur sable (dior), de texture sableuse avec 90 à $95 \%$ de sables totaux, de très faibles taux de carbone $(0,2 \%$ en moyenne), d'azote total $(0,15$ à $1 \%)$ et de bases échangeables (somme totale de $1,77 \mathrm{meq} / 100 \mathrm{~g}$ ). Le $\mathrm{pH}$ est légèrement acide de 5,5 à 6,5.

Les sols de la vallée du Sénégal sont de type vertisols, très argileux (45 à $60 \%$ ), battants en surface avec une forte capacité de rétention en eau, une faible teneur en matière organique $(0,5 \%)$, un $\mathrm{pH}$ faiblement acide et une capacité d'échanges moyenne (20 à $30 \mathrm{meq} / 100 \mathrm{~g})$.

\subsection{Variabilité des pratiques entre agriculture conventionnelle et «agriculture saine et durable » en chou pommé et tomate dans les Niayes}

Des parcelles maraîchères paysannes cultivées suivant les règles d'ENDA-PRONAT ont été comparées avec des parcelles cultivées en conventionnel pour quantifier les différences de rendement. Les suivis de parcelles ont été mis en place pour deux cultures maraîchères : le chou pommé et la tomate, en saison des pluies dans la zone des Niayes en
2016. Toutes les cultures sont arrosées avec un système de goutte-à-goutte.

Pour le chou pommé, 12 parcelles en «agriculture saine et durable» $(\mathrm{AB})$ et 12 parcelles en agriculture conventionnelle (AC) ont été suivies pour faire des comparaisons deux à deux. Pour la tomate, ce sont 6 parcelles de chaque type. Ces parcelles sont cultivées très proches l'une de l'autre, parfois côte-à-côte, parfois séparées par une haie. Les cultures ont été réalisées en saison des pluies et début de saison sèche, avec des semis en pépinière entre le 25 mai et le 24 juillet 2016 . Les récoltes ont été faites du 4 septembre au 3 décembre 2016.

Les cultures de tomate ont été faites en saison des pluies et début de saison sèche, avec des semis entre le 12 juillet et le 29 septembre 2016 , et des récoltes entre le 20 octobre 2016 et le 30 janvier 2017. Toutes les parcelles en AC et en AB ont été récoltées.

Les variables suivies sont:

- le précédent cultural: deux catégories pour le chou pommé: 1) jachère, 2) autres (oignon, chou); deux catégories pour la tomate: 1) oignon, 2) autres (tomate, gombo);

- la date de plantation: deux catégories pour le chou pommé 1) avant le 20 juin, 2) après le 20 juin ; trois catégories pour la tomate: 1) avant le 30 juillet, 2) en août, 3) en septembre ;

- la densité de plantation;

- le nombre de désherbages;

- les apports de matière organique ;

- les quantités d'azote, de phosphore et de potassium apportées (les quantités d'azote, de phosphore et de potassium incluent les apports par les matières organiques et les engrais minéraux);

- le nombre de traitements phytosanitaires;

- le rendement.

Compte tenu du faible nombre de parcelles (12 en AC et 6 en $\mathrm{AB}$ pour le chou pommé, et 6 en $\mathrm{AC}$ et $\mathrm{AB}$ pour la tomate), nous utiliserons les tests non-paramétriques de Mann-Whitney pour les variables quantitatives et le test exact de Fisher pour les variables qualitatives.

\subsection{Variabilité des pratiques entre les villages, zones, variétés et années pour la culture d'oignon}

Les rapports d'activité de l'ONG ENDA-PRONAT (ENDA-PRONAT, 2016a) rassemblent des données moyennes collectées auprès des groupements des producteurs, ainsi que des suivis de parcelles de producteurs appliquant les recommandations de leur cahier des charges, avec les données de variétés, productions et superficies. Ces cultures sont donc toutes considérées comme étant en agriculture biologique. Il n'y aura pas de comparaison avec l'agriculture conventionnelle. Les données sont obtenues par des mesures réalisées chez les producteurs, par pesée complète des récoltes ou des pesées partielles. Ces données sont récapitulées agriculteur par agriculteur ou rassemblées par coopérative ou groupement de producteurs. La culture retenue dans ce travail est l'oignon de saison sèche en culture irriguée. Trois jeux de données ont été analysés. 
Tableau 3. Rendements ( $\mathrm{t} / \mathrm{ha}$ ) d'oignon, chou pommé et tomate au Sénégal en agriculture conventionnelle (AC) et agriculture biologique (AB). Table 3. Onion, cabbage, tomato yields (t/ha) in Senegal in conventional agriculture (AC) and organic agriculture (AB).

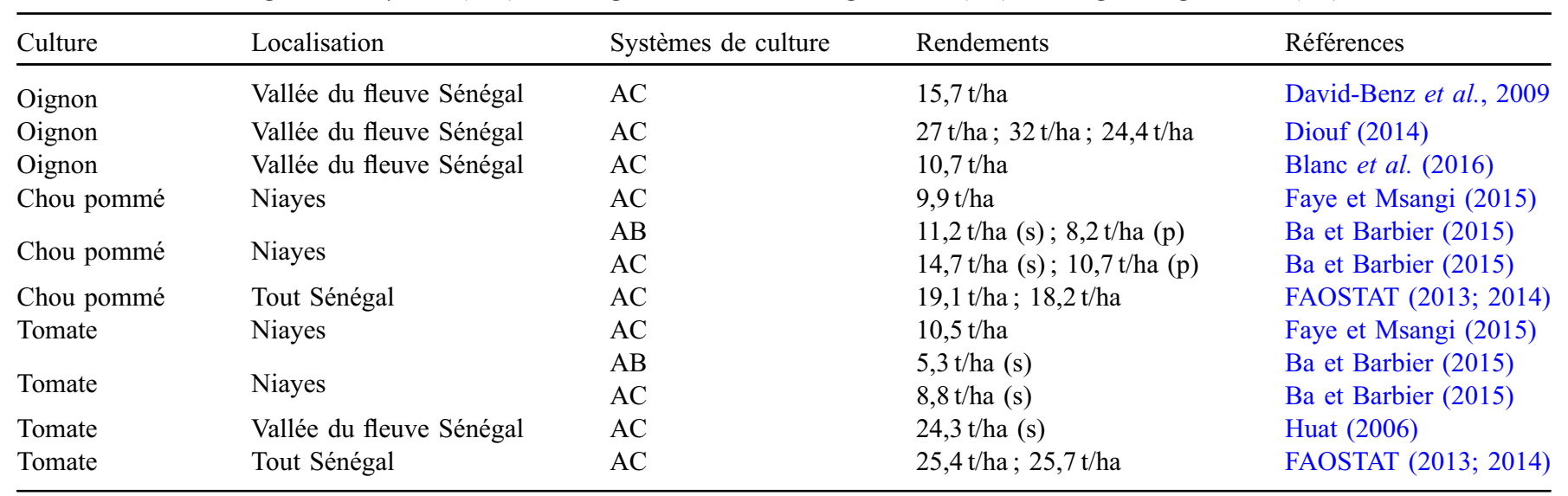

(s) : cultures de saison sèche; (p) : cultures de saison des pluies.

Un premier jeu de données issu des communes de Diouroup et Keur Moussa concerne 3 années de récolte en 2013, 2014 et 2015. Keur Moussa est située dans les Niayes, zone de production maraîchère traditionnelle à $50 \mathrm{~km}$ à l'est de Dakar. Diouroup est située dans le bassin arachidier, à $110 \mathrm{~km}$ au sud de Dakar, où la production d'oignons n'est pas traditionnelle. À Diouroup en 2013, 2014 et 2015, 25 parcelles ont été suivies, ainsi que 39 à Keur Moussa en 2014 et 2015. Les surfaces et rendements sont disponibles, mais non les variétés.

Un deuxième jeu de données de la commune de Keur Moussa présente les récoltes de 2016 et 2017, provenant des saisons de culture 2015-2016 et 2016-2017. Pour la saison 2015-2016, 34 parcelles ont été suivies dans 5 villages autour de Keur Moussa (Yadé, Ndeuye, Niakhip, Keur Moussa, Keur Guilaye), avec les variétés cultivées Galmi, Mercédès, Orient. Pour la saison 2016-2017, 24 parcelles ont été suivies dans 3 villages autour de Keur Moussa (Yadé, Niakhip, Keur Moussa), avec les variétés cultivées Gandiol, Mercédès, Orient, Galmi et Dayo. Les surfaces, rendements, quantité de matière organique et quantité d'azote sont disponibles.

Un troisième jeu de données correspond aux données collectées à Guédé dans la vallée du fleuve Sénégal $(450 \mathrm{~km}$ de Dakar) et auprès de groupements de femmes. Les rendements sont calculés sur les quantités totales récoltées par les 6 groupements (Guédé village, Lérabé, Abdoul Binti, Alpha Dia, Korkadjé, Belel) pour les années 2013, 2014 et 2015. Les données détaillées des cultures ne sont pas disponibles.

Les variables analysées ont été les rendements pour l'ensemble des parcelles, les années, les superficies, les variétés et les apports de matière organique pour les villages de la zone de Keur Moussa. Comme pour la tomate et le chou pommé, les rendements sont mesurés «bord champ», en ne tenant pas compte des pertes après récolte toutes qualités confondues.

La taille des échantillons varie suivant les variables analysées. La variabilité a été analysée avec le test nonparamétrique de Kruskal-Wallis étant donné les effectifs faibles au sein des différentes catégories. Le logiciel XLSTATPremium version 19.6 a été utilisé pour traiter les données.

\section{Résultats et discussion}

\subsection{Revue de littérature}

Peu de données ont été collectées sur les rendements en agriculture biologique du chou pommé, de l'oignon et de la tomate au Sénégal. Les rendements en agriculture conventionnelle sont variables selon les études, allant par exemple de $10,7 \mathrm{t} /$ ha (Blanc et al., 2016) à $32 \mathrm{t} /$ ha (Diouf, 2014) pour l'oignon dans la vallée du Sénégal. Seul un article a considéré les rendements en $\mathrm{AB}$ et a observé des rendements en $\mathrm{AB}$ plus faibles qu'en $\mathrm{AC}$ (Ba et Barbier, 2015) (11,2 t/ha en $\mathrm{AB}$ et $14,7 \mathrm{t} /$ ha en $\mathrm{AC}$ pour le chou pommé ; $5,3 \mathrm{t} /$ ha en $\mathrm{AB}$ et $8,8 \mathrm{t} / \mathrm{ha}$ en $\mathrm{AC}$ pour la tomate) (Tab. 3).

\subsection{Variabilité des pratiques entre agriculture conventionnelle et agriculture biologique pour le chou pommé et la tomate dans les Niayes}

\subsubsection{Chou pommé}

Les résultats concernant le chou pommé sont récapitulés dans le Tableau 4. Toutes les parcelles en AC ont été récoltées alors que seulement 6 sur 12 ont été récoltées en AB. La cause principale d'abandon a été un excès d'eau sur des parcelles mal drainées, accentué par l'absence de fertilisation organique et l'absence d'irrigation à la transplantation. L'analyse statistique a montré que le groupe de parcelles $\mathrm{AC}$ ne se distinguait pas du groupe de parcelle en $\mathrm{AB}$ en termes de précédent cultural, date de plantation, densité de plantation, nombre de désherbages, apport de matière organique, quantités de phosphore et de potassium apportées et rendement. Les seules différences observées concernent le nombre de traitements phytosanitaires (valeur de $p: 0,049$ ) et la quantité d'azote (valeur de $p: 0,002$ ), qui sont supérieurs pour les parcelles en AC. Les différences de pratiques entre les deux systèmes $\mathrm{AB}$ et $\mathrm{AC}$ de ces deux variables sont explicatives.

Les spécialités phytopharmaceutiques utilisées sont le diméthoate, le profénofos (produits de synthèse) et le Bacillus 
Tableau 4. Comparaison des rendements et des itinéraires techniques de parcelles de chou pommé dans les Niayes (Sénégal) en agriculture biologique $(\mathrm{AB})$ et en agriculture conventionnelle $(\mathrm{AC})$.

Table 4. Comparison of yields and technical itineraries of cabbage fields in the Niayes area (Senegal) in organic farming (AB) and conventional agriculture $(A C)$.

\begin{tabular}{|c|c|c|c|c|c|c|c|c|c|c|}
\hline \multirow[b]{2}{*}{$\begin{array}{l}\text { Type } \\
\text { d'itinéraires } \\
\text { techniques } \\
\end{array}$} & \multicolumn{3}{|c|}{$\begin{array}{l}\text { Variables qualitatives (proportion de } \\
\text { parcelles concernées par la catégorie) }\end{array}$} & \multicolumn{7}{|c|}{ Variables quantitatives } \\
\hline & $\begin{array}{l}\text { Précédent } \\
\text { cultural }\end{array}$ & $\begin{array}{l}\text { Date } \\
\text { moyenne } \\
\text { plantation }\end{array}$ & $\begin{array}{l}\text { Apport de } \\
\text { matière } \\
\text { organique }\end{array}$ & $\begin{array}{l}\text { Densité } \\
\text { plantation } \\
\left(\mathrm{plts} / \mathrm{m}^{2}\right)\end{array}$ & $\begin{array}{l}\text { Nombre de } \\
\text { désherbages }\end{array}$ & $\begin{array}{l}\text { Quantité } \\
\text { apportée } \\
\mathrm{N}(\mathrm{kg} / \mathrm{ha})\end{array}$ & $\begin{array}{l}\text { Quantité } \\
\text { apportée } \\
\mathrm{P}_{2} \mathrm{O}_{5}(\mathrm{~kg} / \mathrm{ha})\end{array}$ & $\begin{array}{l}\text { Quantité } \\
\text { apportée } \\
\mathrm{K}(\mathrm{kg} / \mathrm{ha})\end{array}$ & $\begin{array}{l}\text { Nombre de } \\
\text { traitements } \\
\text { phytosanitaires }\end{array}$ & $\begin{array}{l}\text { Rendement } \\
(\mathrm{t} / \mathrm{ha})\end{array}$ \\
\hline $\mathrm{AB}$ & $\begin{array}{l}\text { Jachère } \\
\text { 2/12 (a) } \\
\text { Autres } 1 \\
0 / 12 \text { (a) }\end{array}$ & $\begin{array}{l}\text { Avant } 20 \\
\text { juin } 6 / 12 \text { (a) } \\
\text { Après } 20 \\
\text { juin } 6 / 12 \text { (a) }\end{array}$ & $\begin{array}{l}\text { Apport } \\
3 / 12 \text { (a) }\end{array}$ & $7,9 \pm 4,9$ (a) & $2,5 \pm 1,4$ (a) & $6 \pm 8(a)$ & $5 \pm 6(a)$ & $8 \pm 15$ (a) & 1,0 (a) & $7,0 \pm 2,8$ (a) \\
\hline $\mathrm{AC}$ & $\begin{array}{l}\text { Jachère } \\
1 / 12(a) \\
\text { Autres } \\
11 / 12 \text { (a) }\end{array}$ & $\begin{array}{l}\text { Avant } 20 \\
\text { juin } 3 / 12 \text { (a) } \\
\text { Après } 20 \\
\text { juin } 9 / 12 \text { (a) }\end{array}$ & $\begin{array}{l}\text { Apport } \\
2 / 12 \text { (a) }\end{array}$ & $8,7 \pm 3,4$ (a) & $2,7 \pm 1,0$ (a) & $172 \pm 184(b)$ & $5 \pm 12(\mathrm{a})$ & $2 \pm 6(a)$ & $3,4(b)$ & $9,5 \pm 6,3$ (a) \\
\hline
\end{tabular}

Les valeurs suivies de la même lettre ne sont pas statistiquement significativement différentes.

Tableau 5. Comparaison des rendements et des itinéraires techniques de parcelles de tomates dans les Niayes (Sénégal) en agriculture biologique $(\mathrm{AB})$ et en agriculture conventionnelle (AC).

Table 5. Comparison of yields and technical itineraries of tomato fields in the Niayes area (Senegal) in organic farming (AB) and conventional agriculture $(A C)$.

\begin{tabular}{|c|c|c|c|c|c|c|c|c|c|c|}
\hline \multirow[b]{2}{*}{$\begin{array}{l}\text { Type } \\
\text { d'itinéraires } \\
\text { techniques } \\
\end{array}$} & \multicolumn{3}{|c|}{$\begin{array}{l}\text { Variables qualitatives (proportion de } \\
\text { parcelles concernées par la catégorie) }\end{array}$} & \multicolumn{7}{|c|}{ Variables quantitatives } \\
\hline & $\begin{array}{l}\text { Précédent } \\
\text { cultural }\end{array}$ & $\begin{array}{l}\text { Date } \\
\text { moyenne } \\
\text { plantation }\end{array}$ & $\begin{array}{l}\text { Apport } \\
\text { de matière } \\
\text { organique }\end{array}$ & $\begin{array}{l}\text { Densité } \\
\text { plantation } \\
\left(\mathrm{plts} / \mathrm{m}^{2}\right)\end{array}$ & $\begin{array}{l}\text { Nombre de } \\
\text { désherbages }\end{array}$ & $\begin{array}{l}\text { Quantité } \\
\text { apportée } \\
\mathrm{N}(\mathrm{kg} / \mathrm{ha})\end{array}$ & $\begin{array}{l}\text { Quantité } \\
\text { apportée } \\
\mathrm{P}_{2} \mathrm{O}_{5}(\mathrm{~kg} / \mathrm{ha})\end{array}$ & $\begin{array}{l}\text { Quantité } \\
\text { apportée } \\
\text { K (kg/ha) }\end{array}$ & $\begin{array}{l}\text { Nombre } \\
\text { de traitements } \\
\text { phytosanitaires }\end{array}$ & $\begin{array}{l}\text { Rendement } \\
(\mathrm{t} / \mathrm{ha})\end{array}$ \\
\hline $\mathrm{AB}$ & $\begin{array}{l}\text { Oignon } \\
2 / 6 \text { (a) } \\
\text { Autres } \\
4 / 6 \text { (a) }\end{array}$ & $\begin{array}{l}\text { Avant } \\
15 \text { septembre } \\
2 / 6 \text { (a) } \\
\text { Après } \\
15 \text { septembre } \\
4 / 6 \text { (a) }\end{array}$ & $\begin{array}{l}\text { Apport } \\
0 / 6 \text { (a) }\end{array}$ & $4,0 \pm 0,9$ (a) & $2,0 \pm 0,9$ (a) & $0 \pm 0$ (a) & $0 \pm 0$ (a) & $0 \pm 0$ (a) & $0,3 \pm 0,8$ (a) & $5,1 \pm 2,1$ (a) \\
\hline $\mathrm{AC}$ & $\begin{array}{l}\text { Oignon } \\
6 / 6 \text { (a) } \\
\text { Autres } \\
0 / 6 \text { (b) }\end{array}$ & $\begin{array}{l}\text { Avant } \\
15 \text { septembre } \\
4 / 6 \text { (a) } \\
\text { Après } \\
15 \text { septembre } \\
2 / 6 \text { (a) }\end{array}$ & $\begin{array}{l}\text { Apport } \\
0 / 6 \text { (a) }\end{array}$ & $6,3 \pm 1,0(b)$ & $2,3 \pm 0,5$ (a) & $88 \pm 48(b)$ & $32 \pm 47$ (a) & $17 \pm 20$ (a) & $3,2 \pm 1,2(b)$ & $12,9 \pm 8,8(b)$ \\
\hline Moyenne & & & & $5,2 \pm 1,5$ & $2,2 \pm 0,7$ & $44 \pm 56$ & $16 \pm 36$ & $8 \pm 16$ & $1,8 \pm 1,8$ & $9,0 \pm 7,3$ \\
\hline
\end{tabular}

Les valeurs suivies de la même lettre ne sont pas statistiquement significativement différentes.

thuringiensis (BT), produit biologique. Un seul producteur de chou pommé en $\mathrm{AB}$ a fait des applications d'insecticides (profénofos et BT), alors que les autres producteurs en AB n'ont fait aucun traitement. Deux parcelles ont reçu des traitements à base de bouillon culinaire Mami ${ }^{\circledR}$ avec des résultats contrastés $(1,7$ et $8,4 \mathrm{t} / \mathrm{ha})$. Il n'y a pas de produit biocide ni de répulsif dans ce condiment alimentaire, mais du sucre et de l'amidon qui peuvent être attractifs pour les auxiliaires. Curieusement, ce produit n'a pas été utilisé en agriculture biologique. Les ravageurs observés (Hellula 
Tableau 6. Rendement ( $\mathrm{t} / \mathrm{ha}$ ) de 165 champs observés dans 3 zones de production d'oignon, Diouroup, Keur Moussa et Guédé (Sénégal). Données d'ENDA-PRONAT.

Table 6. Yield of 165 onions fields (t/ha) in 3 production areas: Diouroup, Keur Moussa and Guédé (Senegal). ENDA-PRONAT data.

\begin{tabular}{llll}
\hline Zones & $\begin{array}{l}\text { Nombre de } \\
\text { champs observés }\end{array}$ & $\begin{array}{l}\text { Nombre d'années } \\
\text { observées }\end{array}$ & $\begin{array}{l}\text { Rendement } \\
\text { (t/ha) }\end{array}$ \\
\hline Diouroup & 25 & 3 & $21,0 \pm 9,9$ (a) \\
Keur Mousa & 128 & 3 & $16,8 \pm 8,6(\mathrm{a})$ \\
Guédé & 12 & 3 & $8,6 \pm 4,3$ (b) \\
Valeur de & & & 0,001 \\
$\begin{array}{l}p \text { (unilatérale) } \\
\text { Moyenne }\end{array}$ & & $16,9 \pm 9,7$ \\
\hline
\end{tabular}

Comparaison des moyennes par le test de Kruskal-Wallis à $5 \%$ et comparaison multiples par paires par le test de Dunn.

Les valeurs suivies de la même lettre ne sont pas significativement différentes.

undalis, Plutella xylostella, Chrysodeixis chalcites) sont identiques, quel que soit l'itinéraire technique pratiqué sans que les niveaux de pullulations aient été précisés.

Alors que les 12 parcelles en $\mathrm{AC}$ ont reçu une fertilisation avec des engrais de synthèse (10-10-20, urée), dont 2 avec des apports de fumier de volaille, seulement 3 parcelles en $\mathrm{AB}$ sur les 12 mises en place ont reçu des apports de matière organique (fumier de mouton, fumier de volaille, coques d'arachide). Sur les parcelles ayant bénéficié des apports de fertilisants de synthèse (12 en $\mathrm{AC}$ et 3 en $\mathrm{AB}$ ), la quantité d'azote apportée sur les parcelles $\mathrm{AC}$ est très supérieure à celle apportée en $\mathrm{AB}$ : $172 \mathrm{~kg} / \mathrm{N} / \mathrm{ha}$ contre $14 \mathrm{~kg} / \mathrm{N} / \mathrm{ha}$. La régression linéaire entre les rendements (rdt) et les doses d'azote $(\mathrm{N})$ donne l'équation : $\mathrm{rdt}(\mathrm{t} /$ $\mathrm{ha})=5,9+0,02 \mathrm{~N}(\mathrm{~kg} / \mathrm{ha})\left(r^{2}=0,415\right)$. Cependant, cette analyse prend en compte essentiellement les apports d'azote synthétique. Elle ne permet pas de conclure sur l'efficacité des apports de matière organique. Les quantités apportées de $\mathrm{P}_{2} \mathrm{O}_{5}$ et $\mathrm{K}_{2} \mathrm{O}$ sont très faibles au regard des besoins des cultures de chou pommé.

\subsubsection{Tomate}

Pour les variables qualitatives, les parcelles en $\mathrm{AC}$ et en $\mathrm{AB}$ se distinguent par le précédent cultural (Tab. 5). La proportion de parcelles en $\mathrm{AC}$ avec de l'oignon en précédent cultural est statistiquement supérieure à celle des parcelles en AB. Concernant les variables quantitatives, seuls la densité de plantation, les quantités apportées d'azote, le nombre de traitements phytosanitaires et le rendement sont statistiquement différents entre les parcelles en $\mathrm{AC}$ et en $\mathrm{AB}$. Ainsi, la densité de plantation est en moyenne de 4,0 plants $/ \mathrm{m}^{2}$ en $\mathrm{AB}$ et de 6,3 plants $/ \mathrm{m}^{2}$ en $\mathrm{AC}$ (valeur de $p=0,01$ ). La quantité d'azote apportée est en moyenne de nulle en $\mathrm{AB}$ et de $88 \mathrm{~kg} / \mathrm{ha}$ en $\mathrm{AC}$ (valeur de $p=0,003$ ). La régression linéaire entre les rendements et les doses d'azote donne l'équation: rdt ( $t /$ $\mathrm{ha})=7,1+0,04 \mathrm{~N}(\mathrm{~kg} / \mathrm{ha})\left(r^{2}=0,100\right)$. Comme pour le chou pommé, cette analyse ne porte que sur les doses d'engrais de synthèse apportées sur l'itinéraire AC. Les apports de matière organique ne peuvent donc pas être discutés.

Le nombre de traitements phytosanitaires est en moyenne de 0,3 traitement en $\mathrm{AB}$ et de 3,2 en $\mathrm{AC}$ (valeur de $p=0,006$ ).
Tableau 7. Comparaison interannuelle 2015, 2016 et 2017 des superficies et rendements de 89 champs d'oignons dans la commune de Keur Moussa, dans la zone des Niayes (Sénégal). Données d'ENDA-PRONAT.

Table 7. Inter-annual comparison 2015, 2016, 2017 of areas and yields of 89 onions fields in the Keur Moussa village in Niayes area (Senegal). ENDA-PRONAT data.

\begin{tabular}{llll}
\hline Année & $\begin{array}{l}\text { Nombre de } \\
\text { champs observés }\end{array}$ & $\begin{array}{l}\text { Superficie } \\
\left(\mathrm{m}^{2}\right)\end{array}$ & $\begin{array}{l}\text { Rendement } \\
(\mathrm{t} / \mathrm{ha})\end{array}$ \\
\hline 2015 & 31 & $429 \pm 281$ (a) & $19,4 \pm 8,7$ (a) \\
2016 & 34 & $693 \pm 464(\mathrm{~b})$ & $14,0 \pm 9,6(\mathrm{~b})$ \\
2017 & 24 & $1072 \pm 898$ (b) & $11,0 \pm 7,8$ (b) \\
Valeur de & & 0,001 & 0,001 \\
$p$ unilatérale à 0,05 & & \\
\hline
\end{tabular}

Test de Kruskal-Wallis à $5 \%$ complété par la procédure de comparaisons multiples de Dunn.

Les valeurs suivies de la même lettre ne sont pas significativement différentes.

Les rendements sont en moyenne de 5,1 t/ha en $\mathrm{AB}$ et de $12,9 \mathrm{t} /$ ha en $\mathrm{AC}$ (valeur de $p=0,02$ ). Cette différence peut s'expliquer par l'absence de fertilisation sur les parcelles en $A B$ ainsi que l'absence d'applications de produits phytopharmaceutiques. Les matières actives utilisées sont des insecticides-acaricides de synthèse : diméthoate, imidaclorpride, profénofos, cyhalotrine et dicofol. Chaque agriculteur a sa préférence et n'utilise qu'une seule molécule appliquée 2 à 5 fois pendant la culture. Il n'y a quasiment pas d'application de produits de lutte contre les ravageurs et maladies dans les itinéraires $\mathrm{AB}$ suivis.

\subsection{Variabilité des pratiques entre les villages, zones, variétés et années pour la culture d'oignon}

Compte tenu de la variabilité des données sur l'oignon, seuls les rendements ont été analysés sur les 3 zones identifiées par leurs communes principales: Diouroup, Keur Moussa et Guédé, toutes années confondues. Les rendements obtenus à Guédé $(8,6 \mathrm{t} / \mathrm{ha})$ sont plus faibles que ceux des zones de Diouroup (21,0 t/ha) et Keur Moussa (16,8 t/ha) (Tab. 6). Cette baisse est due à des dégâts de thrips (Thysanoptera $\mathrm{sp}$.), insecte ravageur non maîtrisé par les producteurs.

Les rendements, superficies, variétés et années ont été analysés avec le jeu de données de la commune de Keur Moussa. Le test de Kruskal-Wallis a montré que les superficies des champs en 2015 sont statistiquement plus petites que celles de 2016 et 2017 (valeur de $p=0,001$ ), alors que les rendements sont plus élevés (Tab. 7). Les rendements plus faibles en 2016 et 2017 seraient principalement dus à des attaques de thrips, accentuées par les fortes chaleurs et des méthodes de protection peu efficaces.

Aucune différence de rendement entre les variétés n'a été observée, ni entre les quantités de matière organique (Tab. 8). Le deuxième jeu de données sur la commune de Keur Moussa a permis d'analyser les relations entre apports de matière organique et rendement. Les matières organiques ont été apportées en deux fois : avant la culture et après la transplantation, sous forme de fumier de volaille, bovin ou mouton. Le 
Tableau 8. Comparaison des variétés d'oignons dans la commune de Keur Moussa, dans la zone des Niayes (Sénégal) et apports de matière organique par variété sur 58 champs. Données d'ENDA-PRONAT.

Table 8. Onion varieties comparison in the Keur Moussa village in Niayes area (Senegal) and organic matter supplies on 58 fields. ENDAPRONAT data.

\begin{tabular}{lcll}
\hline Variétés & Nombre de champs & Rendement (t/ha) & Apport matière organique (t/ha) \\
\hline Galmi - Dayo & 8 & $11,3 \pm 4,8(\mathrm{a})$ & $13 \pm 9(\mathrm{a})$ \\
Gandiol & 16 & $12,3 \pm 9,0(\mathrm{a})$ & $12 \pm 9(\mathrm{a})$ \\
Mercédès & 8 & $14,4 \pm 8,7(\mathrm{a})$ & $16 \pm 20(\mathrm{a})$ \\
Orient & 26 & $13,0 \pm 10,3$ (a) & $16 \pm 16(\mathrm{a})$ \\
Valeur de $p$ unilatérale à̀ 0,05 & & 0,58 & 0,76 \\
Moyenne & & $12,8 \pm 9,0$ & $15 \pm 14$ \\
\hline
\end{tabular}

Test de Kruskal-Wallis sur 58 champs, à $5 \%$, complété par la procédure de comparaisons multiples de Dunn. Les valeurs suivies de la même lettre ne sont pas significativement différentes. Vu leurs faibles effectifs, les champs cultivés avec les variétés Galmi et Dayo ont été analysés ensemble pour pouvoir réaliser l'analyse statistique.

fumier de volaille a été utilisé dans $92 \%$ des cas pour le premier apport et $96 \%$ pour le deuxième apport. Les doses ont été calculées à partir du nombre de sacs apportés sur les parcelles et du poids moyen de chaque sac. Ces matières organiques ne sont pas compostées. Les quantités apportées par les agriculteurs sont inférieures aux recommandations des techniciens d'ENDAPRONAT: $15 \pm 14 \mathrm{t} / \mathrm{ha}$, au lieu de $45 \mathrm{t} / \mathrm{ha}$. Le calcul de la régression du rendement en $\mathrm{t} / \mathrm{ha}(\mathrm{Rdt})$ en fonction des apports de matière organique en quantités brutes $(\mathrm{MO})\left(\mathrm{kg} / \mathrm{m}^{2}\right)$ met en évidence un très faible effet des apports de matière organique sur le rendement. Le modèle calculé s'écrit : $\mathrm{Rdt}=10,1+1,8 \times \mathrm{MO}$, avec $r^{2}=0,08$. Cette quasi absence d'effet peut s'expliquer par des apports faibles en quantité et en qualité (matières organiques non compostées), ainsi que par le fait que de nombreux autres facteurs influent sur le rendement.

L'importance des écarts-types montrent de grandes variations de rendement à l'intérieur des différentes catégories constituées pour l'analyse (zones, années, superficies, variétés, apports de matière organique). Cette hétérogénéité traduit la diversité des pratiques des agriculteurs, notamment dans ces zones où la culture se fait par irrigation, la plupart du temps manuelle.

\subsection{Des pratiques à consolider pour renforcer les rendements}

Les rendements de ces trois cultures maraîchères - chou pommé, tomate et oignon - obtenus au Sénégal sont très hétérogènes selon les travaux cités et les publications.

Les rendements en oignon $\mathrm{AB}$ obtenus dans les programmes d'ENDA-PRONAT varient en moyenne de 8,6 à $21,0 \mathrm{t} / \mathrm{ha}$, pour une moyenne de $16,9 \mathrm{t} / \mathrm{ha}$. Ils sont, selon les sources, légèrement supérieurs aux données de la littérature pour les systèmes conventionnels: $107 \%$ des $15,7 \mathrm{t} /$ ha de David-Benz et al. (2009); ou inférieurs : 69 à $53 \%$ des 24,4 t/ ha à $32 \mathrm{t} /$ ha de Diouf (2014). Spécifiquement pour la vallée du Sénégal, le rendement obtenu à Guédé en $\mathrm{AB}(8,6 \mathrm{t} / \mathrm{ha})$ est de $80 \%$ de celui en AC (Blanc et al., 2016).

Les ratios $\mathrm{AB} / \mathrm{AC}$ des suivis comparatifs sont: $74 \%$ pour le chou pommé et $40 \%$ pour la tomate (Tabs. 4 et 5 ). Les données de même nature au Sénégal sont rares : $79 \%$ et $48 \%$ sur chou (Ba et Barbier, 2015) ; $60 \%$ sur tomate (Ba et Barbier, 2015), avec des niveaux de rendement équivalent à ceux de nos suivis de parcelles. Ce sont des baisses supérieures à celles des moyennes des méta-analyses. Lesur-Dumoulin et al. (2017) calculent des ratios moyens de $0,83[0,77-0,90]$ et $0,76[0,65-$ $0,85]$ suivant les modèles utilisés, pour les cultures marâ̂chères.

Les sources des baisses de rendement identifiées dans la littérature sont: les quantités d'azote apportées, le $\mathrm{pH}$ du sol, la nature de l'engrais organique, les meilleures pratiques, la certification, le temps depuis la conversion, l'irrigation, les rotations culturales, les systèmes de polyculture.

Certains de ces facteurs n'ont pas pu être mesurés dans le présent travail : l'irrigation, le $\mathrm{pH}$ du sol, la polyculture, le temps depuis la conversion. D'autres facteurs comme les meilleures pratiques, les engrais organiques et la certification méritent des commentaires au regard de la situation sénégalaise.

L'absence de la mention des applications de pesticides «bio» ou synthétiques dans la littérature de pays du Nord s'explique par le fait que, dans le cadre des agricultures biologiques certifiées par tierce partie, l'utilisation de produits de synthèse $\mathrm{y}$ est nécessairement interdite et respectée. Les différences de pratiques observées entre $\mathrm{AB}$ et $\mathrm{AC}$ dans ce travail relèvent, d'une part, de l'application imparfaite d'un cahier des charges, et d'autre part, d'itinéraires techniques peu standardisés.

Ainsi, les différences observées pour le chou pommé et la tomate portent sur le nombre de traitements phytosanitaires et les quantités d'azote; d'autres différences apparaissent pour la densité de plantation, le précédent cultural et la taille des parcelles (Tabs. 4 et 5). Si le nombre de traitements phytosanitaires varie entre $\mathrm{AB}$ et $\mathrm{AC}$, l'utilisation des biopesticides n'est pas systématique. Ainsi, la pratique AB, pour certains agriculteurs sénégalais, exclut l'application de tout pesticide. Pour un autre, en cas de nécessité, l'application d'un pesticide de synthèse est possible. Les pratiques de protection des cultures acceptables en agriculture biologique sont peu connues et peu développées. Les producteurs ont des difficultés à identifier les produits commerciaux qui peuvent être utilisés en AB (cas du Bacillus thuringensis sur chou). Les mesures prophylactiques sont peu identifiées et manquent de références spécifiques aux cultures dans les conditions du Sénégal. Les impacts sur le rendement commercial sont importants, comme le montrent les données obtenues sur l'oignon. 
ENDA-PRONAT recommande aux agriculteurs d'utiliser la matière organique. Dans le cas des parcelles suivies dans ce travail, les matières organiques sont d'origine animale. Ces matières organiques se dégradent en quelques mois et ne permettent pas d'augmenter de façon durable le stock d'éléments nutritifs. Il faut donc renouveler régulièrement, à chaque culture, ces apports de matière organique, d'autant plus que les apports sont souvent inférieurs aux recommandations calculées sur la base d'analyses chimiques. Dans les Niayes, si des apports de matière organique sont effectués sur les cultures d'oignon, les cultures de chou et de tomate en saison des pluies, très spéculatives pour les agriculteurs, reçoivent très peu de fertilisation. Cependant, l'absence de corrélation entre quantités de matière organique et rendement sur les cultures d'oignon en $\mathrm{AB}$ montre que les apports de matière organique, nécessaires et à accroître, ne seront pas le seul facteur permettant d'améliorer les rendements.

Ces deux catégories de pratiques, fertilisation et gestion des ravageurs et maladies, qui sont discriminantes pour l'agriculture biologique, ne sont pas bien adoptées par les producteurs suivis dans ce travail.

\section{Conclusion}

La comparaison directe des rendements entre agriculture biologique et agriculture conventionnelle sur la base de ces données donne quelques indications. Les rendements en agriculture biologique obtenus dans les programmes d'ENDAPRONAT sont très variables. Les moyennes observées sont parfois supérieures aux données de la littérature dans les systèmes conventionnels. Cette hétérogénéité des données (champ, périmètres, agriculteurs, parcelles élémentaires) traduit les difficultés à diffuser ces techniques. Mais ces observations vont dans le même sens que le résultat obtenu par Lesur-Dumoulin et al. (2017), indiquant que pour les cultures horticoles, il y a $20 \%$ de chances d'obtenir des rendements plus élevés en agriculture biologique comparativement aux systèmes conventionnels.

Malgré l'hétérogénéité des rendements, les pratiques recommandées par ENDA-PRONAT et son encadrement apparaissent suffisamment robustes pour être développées par les agriculteurs. Accompagné par la mise en place d'un système de commercialisation participatif en construction (Tankam, 2017), le mode de production biologique est une voie réaliste pour le développement de l'agriculture dans ces contextes.

Remerciements. Nous tenons à remercier le méta-programme Glofoods de l'INRA et du CIRAD, qui a permis de réaliser ce programme sur le terrain au Sénégal, tous les intervenants de l'ONG ENDA-PRONAT qui ont mis à disposition leurs réseaux, leurs documents et leurs compétences, ainsi que les agriculteurs qui ont bien voulu accueillir les stagiaires et fournir les renseignements souhaités. Enfin, un grand merci au Dr Marion Robert pour son aide précieuse et efficace pour les traitements statistiques des nombreuses données.

\section{Références}

Ba AB, Barbier B. 2015. Economic and environmental performances of organic farming system compared to conventional farming system: a case farm model to simulate the horticultural sector of the Niayes region in Senegal. J Horticult 2(4): 152. DOI: 10.4172/ 2376-0354.1000152.

Blanc E, Lepine A, Strobl E. 2016. Determinants of crop yield and profit for family farms: evidence from the Senegal River Valley. Expl Agric 52(1): 110-136. DOI: 10.1017/S0014479714000581.

David-Benz H, Diop M, Fall C, Wade I. 2009. Oignon: une production en plein essor pour répondre à la demande urbaine. In: Duteurtre G, Faye MD, Dieye PN, eds. L'agriculture sénégalaise à l'épreuve du marché. Paris: ISRA Karthala; pp. 171-196.

Diouf MM. 2014. La filière oignon au Sénégal. Bulletin Analyse économique, CGER Vallée, Saint-Louis-du-Sénégal, Sénégal, 11 p.

ENDA-PRONAT 2016a. Programme agriculture saine et durable. Rapport annuel 2015 et prolongation 2016. Projet BMZ P 2011.3426.1 «Génération des revenus à travers la protection communautaire des ressources naturelles dans 5 zones écologiques du Sénégal ». Dakar, Sénégal, 90 p.

ENDA-PRONAT. 2016b. Fiches de capitalisation sur l'agriculture écologique et biologique au Sénégal. Dakar, Sénégal, 46 p. http:// www.endapronat.org/images/fiches\%20aeb\%20recherches\% 20paysannes.compressed.pdf.

FAOSTAT. [ 2017/07/18]. http://www.fao.org/faostat/en/\#data/QC.

Faye A, Msangi S. 2015. Optimal groundwater management under uncertain climate and its implication on irrigation water availability in the coastal North-Niayes region of Senegal. ECOMOD-2015, 15-17 July, Boston College.

Huat J. 2006. Facteurs limitatifs du rendement de la tomate industrielle en périmètres irrigués au Nord Sénégal. Cahiers Agricultures 15(3): 293-300.

Kirchmann H, Kätterer T, Bergström L, Börjesson G, Bolinder MA. 2016. Flaws and criteria for design and evaluation of comparative organic and conventional cropping systems. Field Crops Res 186: 99-106. DOI: 10.1016/j.fcr.2015.11.006.

Lesur-Dumoulin C, Malézieux E, Ben-Ari T, Langlais C, Makowski D. 2017. Lower average yields but similar variability in organic versus conventional horticulture. A meta-analysis. Agron Sustain Dev 37-45. DOI: 10.1007/s13593-017-0455-5.

Ponisio LC, M'Gonigle LK, Mace KC, Palomino J, de Valpine P, Kremen C. 2015. Diversification practices reduce organic to conventional yield gap. Proc $R$ Soc $B$ 282: 20141396. DOI: 10.1098/rpsb.2014.1396.

Seufert V, Ramankutty N, Foley AJ. 2012. Comparing the yields of organic and conventional agriculture. Nature 485: 229-232. DOI: 10.1038 /nature 11069 .

Tankam C. 2017. L'organisation des marchés de producteurs des fruits et légumes biologiques à Nairobi, Kenya. Cahiers Agricultures 26; 35006. DOI: 10.1051/cagri/2017020.

Thorup-Kristensen K, Dresbøll DB, Kristensen HL. 2012. Crop yield, root growth, and nutrient dynamics in a conventional and three organic cropping systems with different levels of external inputs and $\mathrm{N}$ re-cycling through fertility building crops. Europ $J$ Agronomy 37: 66-82. DOI: 10.1016/j.eja2011.11.004.

Citation de l'article : de Bon H, Brun-Diallo L, Sène J-M, Simon S, Sow MA. 2019. Rendements et pratiques des cultures maraîchères en agriculture biologique au Sénégal. Cah. Agric. 28: 2. 